\title{
Influence of nonequilibrium phonons on the amplitude of magnetoquantum oscillations in the point-contact resistance
}

\author{
N.L. Bobrov ${ }^{a, b}$, J.A. Kokkedee ${ }^{a}$, N.N. Gribov ${ }^{a, b}$, I.K. Yanson ${ }^{b}$, A.G.M. Jansen ${ }^{a}$ \\ ${ }^{a}$ High Magnetic Field Laboratory, \\ Max-Planck-Institut für Festkörperforschung and Centre National de la Recherche Scientifique, \\ 25 avenue des Martyrs, BP 166, \\ F-38042 Grenoble CEDEX 09, France \\ ${ }^{b}$ Institute for Low Temperature Physics and Engineering of the Ukrainian Academy of Sciences, \\ 47 Lenin avenue, 310164 Kharkov, Ukraine \\ Email address: bobrov@ilt.kharkov.ua
}

(Dated: April 25, 2022; Published Physica B, 204, 83 (1995))

\begin{abstract}
For metallic point contacts with $\mathrm{Be}$ and $\mathrm{Al}$ the magnetoquantum oscillations in the contact resistance have been investigated as a function of the applied voltage over the contact. For one set of point contacts the oscillation amplitude is found to vary nonmonotonously with the applied voltage with similarities to the point-contact spectrum of the electron-phonon interaction. The other part of the investigated point contacts shows a decrease of the oscillation amplitude with increasing bias voltage. For the understanding of the voltage dependence of the amplitude of the point-contact magnetoresistance oscillations the influence of nonequilibrium phonons generated by the ballistically injected electrons will be discussed.

PACS numbers: 71.38.-k, 73.40.Jn, 74.25.Kc, 74.45.+c
\end{abstract}

\section{INTRODUCTION}

In a magnetic field $B$ the permitted electronic states in $k$-space are located on the so-called Landau tubes. The cross-sections of these tubes are determined by the condition of area quantization in a plane perpendicular to the magnetic field direction. If one considers the tube with the largest area of cross-section which is still partially inside the Fermi surface (FS), its occupied part will shrink as $B$ increases and vanishes with infinite rate at the moment the tube touches the FS. Such an abrupt decrease of the occupation occurs periodically in the inverse field $1 / \mathrm{B}$ causing the oscillation of free energy and magnetization (de Haas-van Alphen effect) as $B$ changes. The frequency $F$ of such oscillations is given by the OnsagerLifshitz relation

$$
F=(\hbar / 2 \pi e) A
$$

where $A$ is the extremal area of the FS cross-section.

Blurring the boundary between occupied and unoccupied states at finite temperature $T$ reduces the amplitude of the oscillations. The corresponding reduction factor is

$$
R_{T}=\frac{2 \pi^{2} n k_{B} T / \hbar \omega_{c}}{\operatorname{sh}\left(2 \pi^{2} n k_{B} T / \hbar \omega_{c}\right)} ;
$$

where $n$ is the harmonic number of the oscillations and $\omega_{c}=e B / m$ the cyclotron frequency. Furthermore, due to elastic scattering on the impurities the momentum relaxation time of the electrons becomes finite. This leads to the broadening of the Landau levels and accordingly decreases the amplitude nearly in the same way as if the temperature rises from the actual value $T$ to $T_{\text {eff }}=T+x$. The corresponding reduction factor (named Dingle factor) has the form

$$
R_{D}=\exp \left(-2 \pi^{2} k_{B} n x / \hbar \omega_{c}\right)
$$

where $x=\hbar / 2 \pi k_{B} \tau$ is known as the Dingle temperature. As it follows from the theory for noninteracting particles, the contribution to the Dingle temperature results from any electron scattering process including electron phonon collisions [1]. However, with many-body effects taken into account, the scattering of electrons on phonons seems not to influence the Dingle temperature [1]. For rising temperatures, the additional electron scattering by phonons is compensated by the decrease of effective mass $m$ * approaching the "bare" mass value $m$. This is due to the fact that the reduction factor $R_{T}$ for the temperature dependence keeps almost exactly the same form but the "bare" mass $m$ of the electrons in Eq.(2) should be replaced by the effective mass $m^{*}(T)=m(1+\lambda(T))$ renormalized for the electron-phonon interaction $(\lambda$ is the electron-phonon interaction (EPI) parameter). Although the EPI renormalizes the cyclotron mass which 
determines the reduction of amplitude with temperature, it has no influence on the mass value involved into the Dingle factor $R_{D}$ related to collision broadening, i.e. the "bare" mass $m$ has to be taken into expression (3) [1].

In traditional experiments of magnetic oscillations the investigation of the effect of the electron-phonon interaction on the amplitude of the oscillations is only possible for a limited number of metals with a low Debye temperature (e.g. $\mathrm{Hg}$ ), where phonons may be excited at temperatures low enough for the oscillations to be observed. Point contacts with the size of tens or hundreds of angstroems present a unique opportunity to study the effect of nonequilibrium phonons generated in the contact on the amplitude of the magnetic oscillations. The simplest model of the contact is a circular orifice in a thin nontransparent screen, separating two metal half-spaces. If the contact size (diameter $d$ ) is small compared to the inelastic mean free path $l_{e}$ of the electrons, a voltage $V$ applied over the contact yields a nonequilibrium distribution of the electrons with a well-defined energy $\mathrm{eV}$. To put it differently, the electrons are being divided into two groups with a difference $e V$ in the Fermi level exactly given by the applied voltage [2], In this case the electrons with an excess energy can make a transition to a lower energy emitting one phonon. The multi-phonon emission processes also take place, but their probability is much less. These emission processes lead to a voltagedependent increase in the contact resistance containing detailed information on the energy dependence of the inelastic scattering of the electrons [3]. The experimental study of nonlinear current-voltage characteristic of point-contacts (a technique known as point-contact spectroscopy) has been successfully applied for the direct determination of the Eliashberg function $\alpha^{2} F(\omega)$ for the electron-phonon interaction.

Thus, by the bias applied across the contact, the nonequilibrium phonons are generated in the contact area with any possible value of energy up to $\mathrm{eV}$. It is of a great importance to note that, due to $l_{e}>d$, only a small fraction of the total power is released in the direct vicinity of the contact area and the temperature of the contacting banks of the contact remains practically equal to that of the helium bath. With the used technique with mechanical adjustment of the point contact the elastic mean free path $l_{i}$ of the electrons in the construction area is of the same order as the contact size $d$ which is much less than the mean free path in the electrode. In this case the contact resistance for zero bias in the model of a circular orifice is determined by the Wexler formula [4]

$$
R_{0}=\frac{16 \rho l}{3 \pi d^{2}}+\beta\left(l_{i} / d\right) \frac{\rho}{d},
$$

where $\rho l=p_{F} / e^{2} n_{0}$ is the product of resistivity $\rho$ and mean free path $l$ of the electrons, constant for a given material; $p_{F}$ the Fermi momentum and $n_{0}$ the charge carrier density, $\beta\left(l_{i} / d\right)$ in Eq.(4) is a monotonously decreasing function of $l_{i} / d$ with $\beta(0)=1$ and $\beta(\infty)=9 \pi^{2} / 128$. In this expression the first term describes the Sharvin component of the point-contact resistance, which is independent of the electron mean free path and is determined by the topology of the FS for a given metal. The second term is the Maxwell component of the pointcontact $(\mathrm{PC})$ resistance which depends on the metal purity in the constriction area. When a magnetic field is applied to a point its resistance shows an oscillating component as a result of the quantization of the electron energy spectrum $[5,6]$. The oscillations of the $\mathrm{PC}$ resistance have its origin in both the Maxwell and the Sharvin part of the resistance, depending on the parameters of the metal.

In the contacts with usual metals the Larmor radius $r_{\mathrm{B}}$ of the electrons in fields up to $20 \mathrm{~T}$ exceeds the typical contact diameters (for instance, for berilium only for the largest contacts with $d \approx 500 \AA$ and for $B=10 \mathrm{~T}$, corresponding to $r_{\mathrm{B}}=m^{*} v_{F} / e B=3000 \AA$, the quantities become comparable). Therefore, the magnetoquantum oscillations in the point-contact resistance are mainly the result of quantizing conditions in the neighbouring banks near the constriction. The oscillations in the density of states yield oscillations in the Sharvin component of point-contact resistance. This constitutes an essential difference with the observed oscillations of the magnetoresistance in semimetal point contacts [5] (where the Maxwell component of the resistance oscillates), and the ordinary Shubnikov-de Haas ( $\mathrm{SdH}$ ) effect in bulk conductors, caused by quantum oscillations of the collosion integral [1].

It is quite surprising that the influence of impurities in the constriction area is opposite to the usual dependence as expressed by the Dingle reduction factor for bulk material. When the magnetic field is applied parallel to the contact axis impurities in the contact area increase the amplitude of the magnetoresistance oscillations $[7,8]$. The contribution to the magnetoquantum oscillations comes mainly from the areas of extremal crosssection of the FS perpendicular to $p_{z}$ (the $z$-axis is parallel both to the magnetic field direction and the point-contact axis). The small value of the velocity component $v_{z}$ of the 


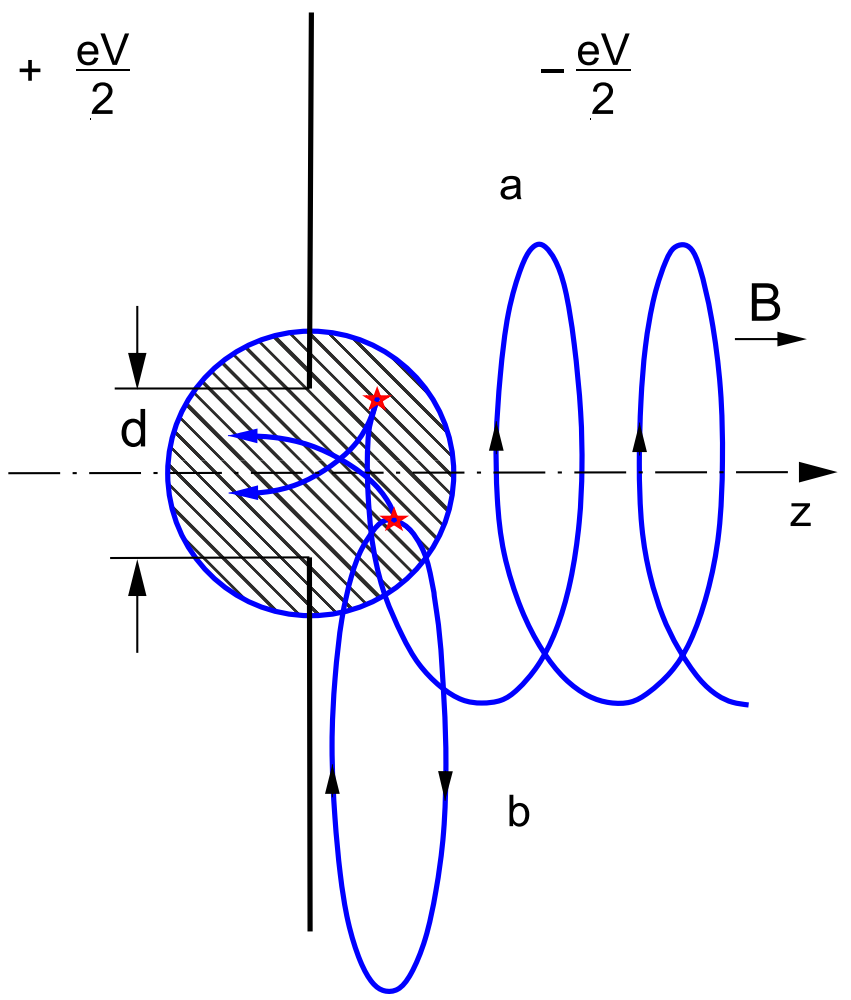

FIG. 1: Scattering of electrons moving along the quantized orbits, by the nonequilibrium phonons (denoted by stars) accumulated near the contact. In a scattering event an electron changes its momentum along $z$ with on average a more effective contribution to the current, for initial states with a small negative $v_{z}$-component (a) and a zero $v_{z}$-component (b).

charge carrier transport through the ballistic constriction yields only quite small oscillations in the Sharvin component of the resistance. In the presence of elastic scatterers in the contact area the momenta of the electrons get an isotropic distribution of directions (Fig.1), leading to an effective increase of the $z$-component of the transport velocity for the electrons on extremal cross-sections and correspondingly to an increase of the magnetoquantum oscillations in the Sharvin component of PC resistance. The presence of elastic scatterers in neighbouring banks causes the increase of the Dingle temperature and thus the decrease of the oscillation amplitude.

The first experiments on the investigation of the effect of nonequilibrium phonons on the amplitude of pointcontact magnetoresistance oscillations are described in Refs. $[5,6]$. In the discussion of the obtained experimental results in Ref.[5] only overheating of the electron gas in the contact area was considered while in Ref.[6] two alternative mechanisms of damping were proposed, namely the Dingle temperature rise due to electron-phonon scat- tering and the Joule heating of the contact area.

In the present work we report on the experimental study of the influence of nonequilibrium phonons on the quantum oscillations in $\mathrm{Be}$ and $\mathrm{Al}$ point contacts. These metals were chosen because of the presence of electron pockets with small effective masses which allows to observe the oscillations in rather weak magnetic fields. It should be marked that in the point-contact experiments the metallic contact is used both for the observation of magnetoquantum oscillations in the resistance and for the generation of nonequilibrium phonons by means of an applied voltage over the contact.

\section{SAMPLES AND EXPERIMENTAL DETAILS}

The studied point contacts of $\mathrm{Al}$ and $\mathrm{Be}$ were formed between the edges of two single crystal electrodes with the same orientation. In the case of Be the contact axis coincided with the crystallographic $c$-axis while for $\mathrm{Al}$ it was parallel to the (110)-axis. The applied magnetic field $(<10 T)$ was always parallel to the contact axis. The electrodes were chemically polished in order to remove the defect layer resulting from the spark cutting. Both a technique of mechanically shifting the electrodes along the edges [9] and an electroforming method [10] were used for the contact adjustment at liquid helium temperatures. All experiments were done at $1.3 \mathrm{~K}$. The first and second derivatives of the current $(I)$-voltage $(V)$ curves were obtained by conventional modulation techniques with data registration as a function of the applied voltage and magnetic field. The characteristics $d V /\left.d I(V)\right|_{B=\text { const }}$, $d V /\left.d I(B)\right|_{V=\text { const }}$ and $d^{2} V / d I^{2}(V)$ have been measured.

Fig.2 presents an example of the $d^{2} I / d V^{2} \equiv-\left(d^{2} V / d I^{2}\right) /(d V / d I)^{3}$ spectrum of a Be contact of $16.24 \Omega$, the oscillating component of magnetoresistance and its Fourier transformed spectrum. The spectrum shows clearly the structure between 40 and $80 \mathrm{meV}$, which can be related to the $\alpha^{2} F(\omega)$ function for the electron-phonon interaction. The magnetoquantum oscillations studied in the Be resistance correspond to third-band electron surface (cigar) with frequency $985 T$ as known from de Haas-van Alphen experiments [11]. For various bias voltages across the contact the oscillations of $R(B)=d V / d I(B)$ were recorded in the same magnetic field interval (as a rule in the range between 8.5 and $10 \mathrm{~T}$ ). Fig. 3 presents the fragments of such records for two different bias voltages of an $\mathrm{Al}-\mathrm{Al}$ point contact. For the $\mathrm{Al}$ contacts the 

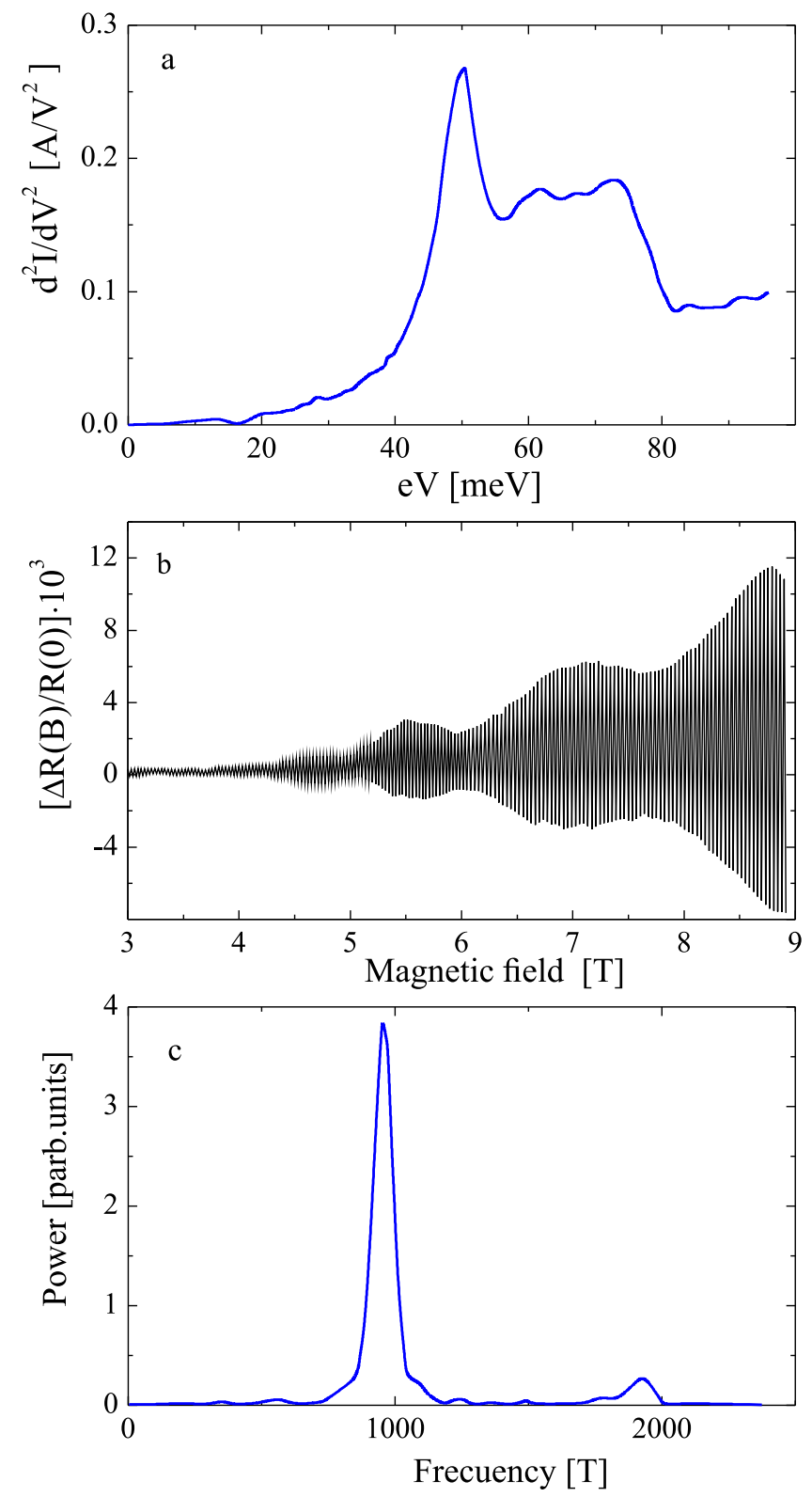

FIG. 2: (a) $d^{2} I / d V^{2}(V)$ spectrum and (b) resistance versus magnetic field for zero bias voltage of a Be point contact of $R=16.24 \Omega$. The magnetic field was oriented parallel to the $c$-axis of beryllium and parallel to the point contact axis. (c) Fourier transform of (b) when plotted against $1 / B$.

investigated oscillation corresponds to the third zone $\gamma$-pocket with frequency $290 T$ [12]. After the recording of the field dependent curves for several bias voltages, the record for $V=0$ was compared with the initial one in order to check the contact stability during the measurements.

Contacts with different resistances (from 0.6 to $20 \Omega$, background levels (from $30 \%$ to $85 \%$ ) in the high voltage

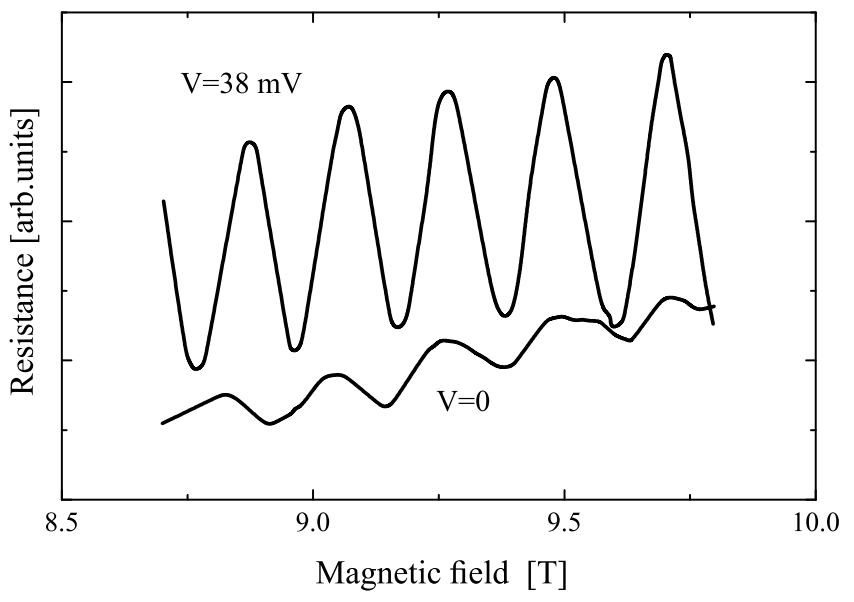

FIG. 3: Fragments of magnetoresistance oscillations for an $\mathrm{Al}$ point contact of $R=141 \Omega$ for two different bias voltages.

part of the $d^{2} I / d V^{2}(V)$ spectrum and degrees of pointcontact spectrum broadening were studied. For metals like $\mathrm{Be}$ and $\mathrm{Al}$ the given resistance values correspond to contact diameters ranging from 50 to $500 \AA$. A full set of characteristics for the voltage and magnetic field dependences could be obtained for five $\mathrm{Al}$ and five Be contacts. In the given magnetic field interval the amplitude of the oscillations was obtained by means of a Fourier analysis. Since the $d V /\left.d I(B)\right|_{V=\text { const. }}$ curves were measured at a constant modulation current while the differential resistance increases with applied bias (increase up to $10 \%$ in the Debye energy range), the obtained amplitudes were normalized for a fixed modulation voltage (the value measured at zero bias). Figs. 4-6 present typical results for the measured contacts. For one group of the investigated point contacts the oscillation amplitude $A_{1}$ of the $\mathrm{PC}$ magnetoresistance increases with increasing bias and its voltage dependence is similar to the point-contact spectrum of the electron-phonon interaction (Figs.4 and 5). Some of the aluminium contacts exhibit a nonmonotonic decrease of the amplitude as a function of voltage (Fig.6). In the following we discuss the possible reasons of such dependencies.

\section{DISCUSSION}

The relaxation of the injected electrons in the pointcontacts leads to the generation of phonons via spontaneous emission processes. The resulting nonequilibrium distribution of the phonons will depend on the applied voltage over the contact. In analogy with the discussed 


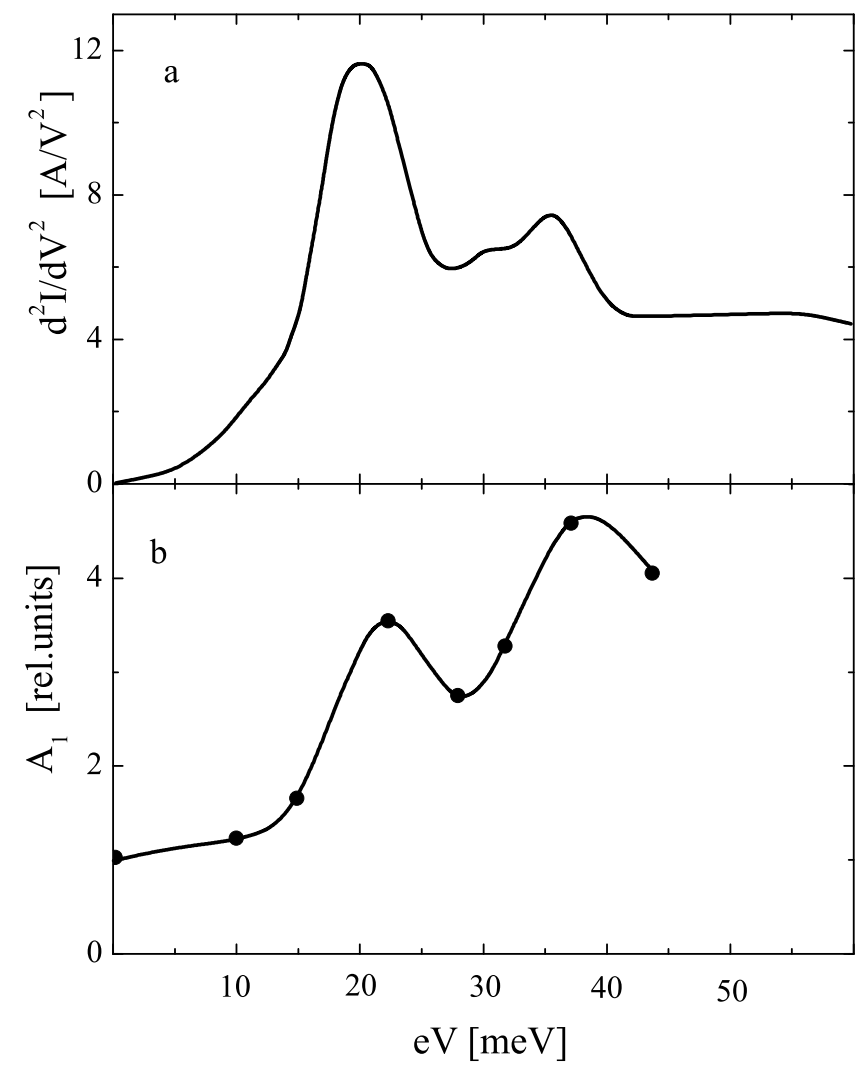

FIG. 4: (a) $d^{2} I / d V^{2}(V)$ spectrum of an $\mathrm{Al}$ point contact of $R=1,41 \Omega$. (b) Relative magnitude of the first harmonic of the point-contact magnetoresistance oscillations versus bias voltage. At $V=0 \mathrm{mV}$ and $B=9.5 T, \Delta R / R_{0} \approx 5 \times 10^{-4}$.

influence of elastic impurity scattering, the scattering of the electrons with these nonequilibrium phonons will influence the amplitude of the magnetoresistance oscillations. As for the elastic scattering, this phonon-induced scattering has a quite different effect on the quantum oscillations for scattering processes in the centre of the contact (with resulting increase of amplitude) compared with scattering in the banks of the constriction (with resulting decrease of amplitude). In the following we will only give a qualitative discussion of the generated phonon distribution and its influence on the magnetoquantum oscillations. Although the elastic mean free path $l_{i}$ of the electrons is of the order of the contact diameter $d$ for mechanically adjusted point contacts, the diffusion length $\Lambda_{e}=\left(l_{i} l_{e}\right)^{1 / 2}$ for inelastic relaxation is mostly larger than the contact dimension. At the Debye energy the ballistic mean free path $l_{e}$ for inelastic scattering equals approximately $3000 \AA$ for aluminium and $1500 \AA$ for beryllium.

Apart from the impurities that have a weak influence

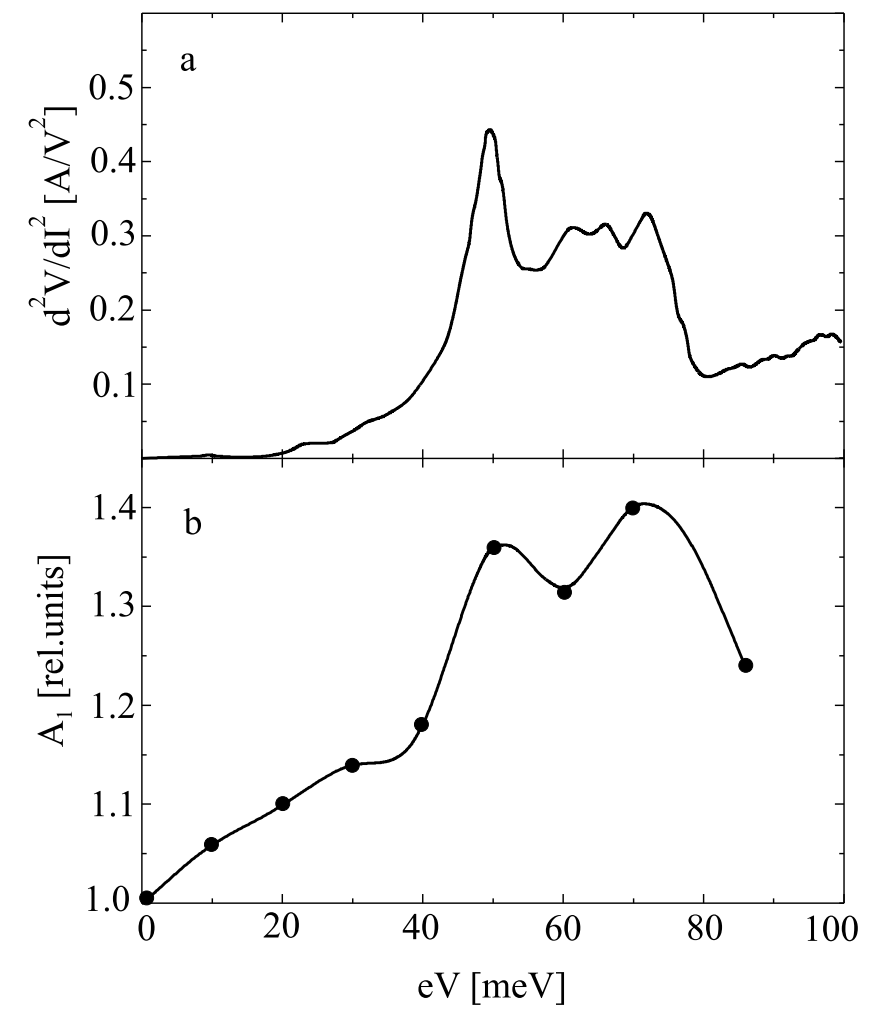

FIG. 5: (a) $d^{2} I / d V^{2}(V)$ spectrum of a Be point contact of $R=8.66 \Omega$. (b) Relative magnitude of the first harmonic of the point-contact magnetoresistance oscillations versus bias voltage. At $V=0 m V$ and $B=9.5 T, \Delta R / R_{0} \approx 2 \times 10^{-3}$.

on the elastic mean free path $l_{i}^{\mathrm{ph}}$ of the phonons although being effective scatterers for the electrons, the pressuretype contacts contain as a rule a great number of lattice defects. These defects have little influence on the elastic mean free path of electrons $l_{i}$, but reduce significantly the elastic mean free path $l_{i}^{\mathrm{ph}}$ of the nonequilibrium phonons generated by the flow of electrons through the constriction [13]. If $l_{i}^{\text {ph }}<d$, the additional electron scattering by the nonequilibrium phonons causes a background in the point-contact spectrum (voltage independent value of the second derivative curves $d^{2} I / d V^{2}(e V)$ at $\left.e V>\hbar \omega_{D}\right)$ comparable with the spectral part of the point-contact spectrum of EPI. In this case a nonequilibrium distribution of phonons is established in the contact with an effective temperature dependent on the position with respect to the contact and on the reabsorbtion coefficient of phonons in the contact area.

The nonequilibrium distribution of the phonons in a point contact differs from Planck's one by the existence of a cut-off at $\hbar \omega_{D}=e V$ (for details see Ref.[13]). In this description of the nonequilibrium 


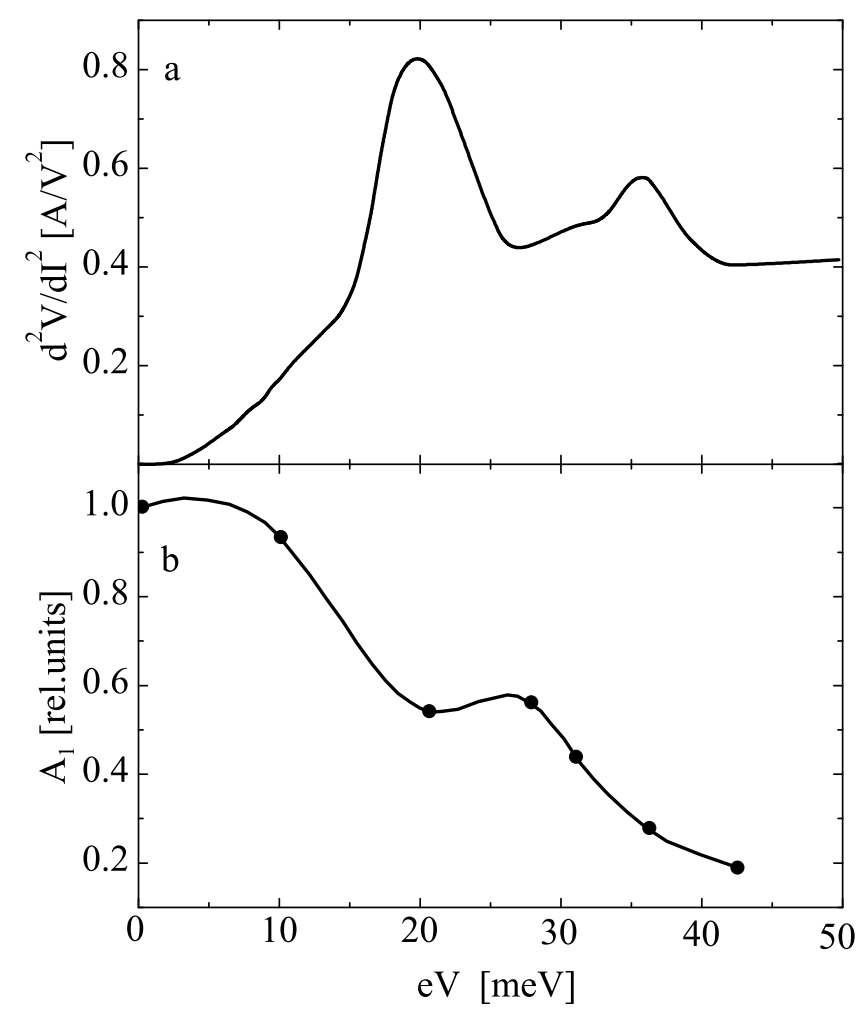

FIG. 6: (a) $d^{2} I / d V^{2}(V)$ spectrum of an Al point contact of $R=8.73 \Omega$ (b) Relative magnitude of the first harmonic of the point-contact magnetoresistance oscillations versus bias voltage. At $V=0 m V$ and $B=9.5 T, \Delta R / R_{0} \approx 3 \times 10^{-3}$.

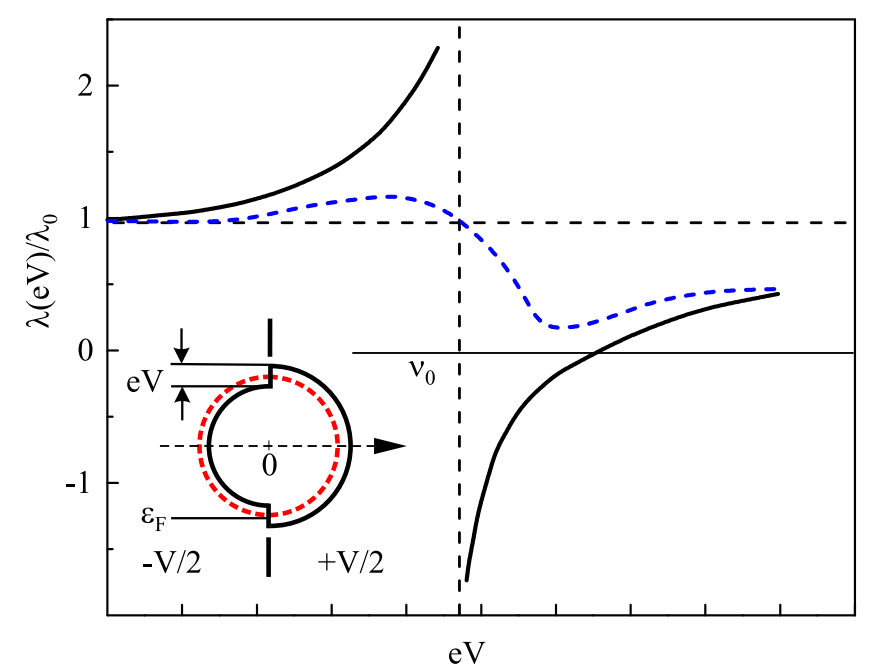

FIG. 7: Schematic representation of theoretically predicted [14] behaviour of the electron-phonon interaction parameter $\lambda$ versus voltage bias near the orifice. Solid and broken lines correspond, respectively, to the infinitesimal small and finite widths of the phonon band at the characteristic energy $e V=\nu_{0}$. The inset shows an electron distribution function of a ballistic point contact in the centre of the orifice. distribution one can define a diffusive mean free path $\Lambda_{e}^{\mathrm{ph}}=\left(l_{i}^{\mathrm{ph}} l_{e}^{\mathrm{ph}}\right)^{1 / 2}\left(\omega_{D} / \omega\right)^{1 / 2}$ for the distance over which the nonequilibrium phonon looses its energy. Here $l_{e}^{\text {ph }}$ is the ballistic inelastic mean free path of the phonons. For Debye energies $l_{e}^{\mathrm{ph}}$ equals by order of magnitude the inelastic mean free path $l_{e}$ of the electrons and has the same energy dependence, i.e. decrease of $l_{e}^{\text {ph }}$ at the characteristic phonon frequencies. The high-frequency phonons having the smallest $\Lambda_{e}^{\mathrm{ph}} / d$ ratio are accumulated near the orifice and strongly interacting with the electrons. The low-frequency phonons (larger $\Lambda_{e}^{\mathrm{ph}} / d$ ratio) dominate in the banks. Assuming the exact analogy with the impurity-scattering, the scattering of the electrons in the contact area causes the randomization of the momenta of the electrons. In this case the current distribution of the $z$-component of the velocity at extremal cross-sections of the Fermi surface increases in a magnetic field leading to an increase of the magnetoquantum oscillations. The electron scattering with generated phonons arriving in the banks leads to a reduction of the amplitude of the oscillations. The resulting voltage dependence of the amplitude of the point-contact magnetoresistance oscillations is determined by the balance between these two processes.

For high Ohmic contacts with aluminium $(R>5 \Omega$, $d<150 \AA)$ the decrease of the oscillation amplitude with increasing bias voltage is always observed. These contacts are small compared to the phonon mean free path and all phonons are leaving for the banks before scattering with electrons in the contact centre takes place. The electron-phonon scattering in the banks reduces the amplitude of the oscillations. For low Ohmic contacts with aluminium the inequality $\Lambda_{e}^{\mathrm{ph}}>d$ is not so rigorous and the phonons accumulate in the contact area. The resulting scattering with electrons in the contact region leads to the observed increase of the oscillation amplitude. One would expect a correlation between the increase of the magnetoresistance oscillations and the occurrence of a pronounced background signal in the second derivative $d^{2} V / d I^{2}(V)$ spectra due to the accumulation of nonequilibrium phonons in the contact area. Unfortunately, we have not seen such a correlation in the data.

As for beryllium, the increase of the amplitude of magnetoresistance oscillations with bias rise was observed for all contacts. It is probably connected with extremely high rigidity of the Be crystal lattice. As a consequence, the deformations formed during contact creation would be stopped near the surface and do not spread to the bulk 
material thus creating efficient reflectors concentrating the phonons in the contact.

The given qualitative model for the voltage dependence of the point-contact oscillations explains only the monotonous part of the voltage dependence but not its similarity to the structure in the EPI spectrum. The phonon energy relaxation length $\Lambda_{\omega}$ will decrease stepwise at voltages corresponding to the phonon frequencies (alike the electron energy relaxation length $\Lambda_{e}$ ). Therefore, the voltage dependence of the amplitude should be expected to be similar to the continuous first derivative curve $d V / d I(V)$. In the following we discuss the energy dependence of the mass-renormalization for the nonequilibrium electrons in a point contact as a possible reason for the nonmonotonous structure observed.

It was predicted in Ref.[14] that the electron mass renormalization parameter $\lambda$ for the effective mass $m^{*}=m(1+\lambda)$ differs from the equilibrium value $\lambda_{0}$ in the vicinity of the orifice. For an applied voltage over a ballistic contact the resulting nonequilibrium distribution of the electrons differs from the spherical Fermi sphere that would give the equilibrium value $\lambda_{0}$ for the electronphonon enhancement factor. For the energy distribution of a ballistic contact (see inset of Fig.7 for this distribution in the centre of the contact) $\lambda$ will depend on the applied voltage. In the centre of the contact one finds [14]

$$
\lambda(e V)=\frac{\lambda_{0}}{2}\left[1+\nu_{0}^{2} /\left(\nu_{0}^{2}-(e V)^{2}\right)\right]
$$

for an Einstein model of the phonon distribution with phonon energy $\nu_{0}$. The expected behaviour of $\lambda(\mathrm{eV})$ is shown schematically in Fig.7. It can be seen that for high voltages the mass renormalization has been reduced by a factor 2 because only half of the Fermi sphere takes part in the mass renormalization for the nonequilibrium distribution in a point contact (see inset of Fig.7). For $\mathrm{eV}=\nu_{0}$ a resonance phenomenon occurs in the mass renormalization. In the same figure we have also given the result for $\lambda(\mathrm{eV})$ for a broadened band of phonon energies around $\nu_{0}$. From the theory can be concluded that the nonmonotonous energy dependence of the effective electron mass at the characteristic phonon frequencies is inherent to the nonequilibrium electron distribution function in point contacts under finite bias condition.

The energy-dependent mass enhancement in a point contact could cause the observed nonmonotonous dependence of the magnetoquantum oscillation amplitude on voltage bias. Note from Fig.7 that the inflection point with negative slope on the $A_{1}(\mathrm{eV})$-curve should be taken as the corresponding characteristic phonon energy. Although the exact position of the maxima in the voltage dependence of the oscillation amplitude changes slightly form contact to contact, the voltage position of these inflection points are usually located at biases larger than the maxima in the phonon density of states. The theory of the voltage-dependent mass enhancement has been evaluated in the absence of a magnetic field [14]. It would be very interesting to include the energy dependence of the effective mass in the theories explaining the observation of magnetoquantum oscillations in ballistic point contacts. We have probably observed such an energy dependence of the effective mass enhancement in the voltage dependence of the oscillation amplitude in a ballistic PC. The ballistic electron transport in a point contact has the advantage to study the energy dependence of the effective mass in the absence of thermal phonon smearing.

\section{CONCLUSION}

In the present work we have studied the voltage dependence of the magnetoquantum oscillation amplitude in the resistance of a metallic point contact in a magnetic field parallel to the contact axis. For the first time it was found that such a dependence shows a nonmonotonous increase with structures similar to the point contact spectrum for the electron-phonon interaction. Such an effect was observed for berilium point contacts as well as for low ohmic aluminium contacts. The possible reason for the increase of the oscillation amplitude may be due to the additional scattering of Landau quantized electrons by phonons generated by the accelerated electrons. These scattering processes of electrons with nonequilibrium phonons enhance the contribution of the charge carrier transport through the contact for the electrons at extremal Landau orbits. Therefore, opposite to the usual suppression of magnetoquantum oscillations by impurity scattering in a bulk metal, the oscillations increase with the increasing scattering at applied voltages. If the scattering with nonequilibrium phonons extends to the banks of the contact the well-known decrease of oscillation amplitude is observed (for high ohmic aluminium contact). The nonmonotonous $\mathrm{eV}$-dependence (i.e. presence of peaks near the maxima in the phonon density of states) is possibly connected with the energy-dependent renormalization of the electron mass in the nonequilib- 
rium distribution of the electrons in a ballistic contact as defined by the applied voltage. for stimulating discussions during the preparation of this paper.

\section{ACKNOWLEDGEMENTS}

The authors would like to thank A. N. Omel'yanchuk, E. N. Bogachek, A. A. Zvyagin and A. D. Dan'kovsky

[1] D. Shoenberg, Magnetic Oscillations in Metals (Cambridge University Press, Cambridge, 1984).

[2] I.O. Kulik, A.N. Omel'yanchuk and R.I. Shekhter, Fiz. Nizk. Temp. 3 (1977) 1543; Sov. J. Low Temp. Phys. 3 (1977) 740 .

[3] I.K. Yanson, Fiz. Nizk. Temp. 9 (1983) 676; Sov. J. Low Temp. Phys. 9 (1983) 343.

[4] G. Wexler, Proc. Phys. Soc. 89 (1966) 927.

[5] N.N. Gribov, O.I. Shklyarevskii, E.I. Ass and V.V. Andrievskii, Fiz. Nizk. Temp. 13 (1987) 642; Sov. J. Low Temp. Phys. 13 (1987) 363.

[6] H.M. Swartjes, A.P. van Gelder, A.G.M. Jansen and P. Wyder, Phys. Rev. B 39 (1989) 3086.

[7] E.M. Bogachek and R.I. Shekhter, Fiz. Nizk. Temp. 14 (1988) 810; Sov. J. Low Temp. Phys. 14 (1988) 445.

[8] O.I. Shklyarevskii, N.N. Gribov and I.K. Yanson, Fiz. Nizk. Temp. 14 (1988) 414; Sov. J. Low Temp. Phys. 14
(1988) 229.

[9] P.N. Chubov, A.I. Akimenko and I.K. Yanson, Fiz. Nizk. Temp. 8 (1982) 64; Sov. J. Low Temp. Phys. 8 (1982) 32.

[10] N.L. Bobrov, L.F. Rybarchenko, V.V. Fisun and I.K. Yanson, Fiz. Nizk. Temp. 13 (1987) 611; Soy. J. Low Temp. Phys. 13 (1987) 344; arXiv:1512.01800.

[11] J.H. Tripp, P.M. Everett, W.L. Gordon and R.W. Stark, Phys. Rev. 180 (1969) 669.

[12] C.O. Larson and W.L. Gordon, Phys. Rev. 156 (1967) 703.

[13] I.O. Kulik, Fiz. Nizk. Temp. 11 (1985) 937; Sov. J. Low Temp. Phys. 11 (1985) 516.

[14] A.N. Omel'yanchuk, Fiz. Nizk. Temp. 11 (1985) 543; Sov. J. Low Temp. Phys. 11 (1985) 298. 